PPPL-2955

UC-427

\title{
GLOBAL STRUCTURES OF ALFVÉN-BALLOONING MODES IN MAGNETOSPHERIC PLASMAS
}

BY

G. VETOULIS AND L. CHEN

MARCH, 1994

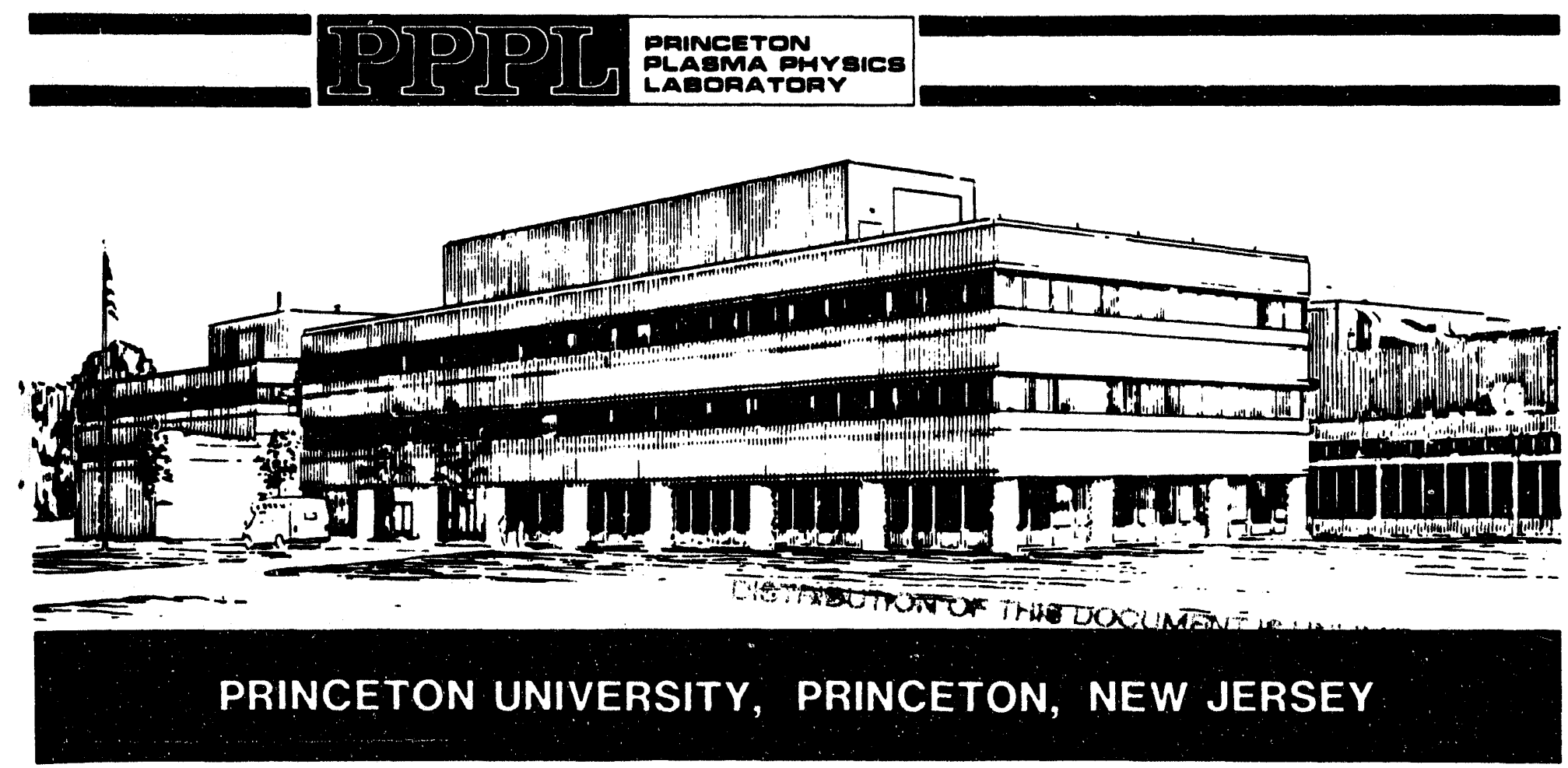




\section{NOTICE}

This report was prepared as an account of work sponsored by an agency of the United States Government. - Neither the United States -Government nor any agency thereof, nor any of their employees, makes any warranty, express or implied, or assumes any legal liability or responsibility for the accuracy, completeness, or usefulness of any information, apparatus, product, or process disclosed, or represents that its use would not infringe privately owned rights. Reference herein to any specific commercial produce, process, or service by trade name, trademark, manufacturer, or otherwise, does not necessarily constitute or imply its endorsement, recommendation, or favoring by the United States Government or any agency thereof. The views and opinions of authors expressed herain do not necessarily state or reflect those of the United States Government oi any agency thereof.

\section{NOTICE}

This report has been reproduced from the best available copy. Available in paper cc py and microfiche.

Number of pages in this report: 13

DOE and DOE contractors can obtain copies of thils report from:

Office of Scientific and Technical Information P.O. Box 62

Oak Ridge, TN 37831;

(615) $576-8401$.

This report is publicly available from the:

National Technical Information Service

Department of Commerce 5285 Port Royal Road

Springfield, Virginia 22161

(703) $487-4650$ 


\title{
Global structures of Alfvén-ballooning modes in magnetospheric plasmas
}

\author{
George Vetoulis and Liu Chen * \\ Princeton Plasma Physics Laboratory \\ P.O. Box 451, Princeton, NJ 08544
}

March 1, 1994

\begin{abstract}
We show that a steep plasma pressure gradient can lead to radially localized Alfvén modes, which are damped through coupling to filed line resonances. These have been called drift Alfvén balloning modes (DABM) by [3] and are the prime candidates to explain Pc4-Pc5 geomagnetic pulsations observed during storms. A strong dependence of the damping rate on the azimuthal wave number $m$ is established, as well as on the equilibrium profile. A minimum azimuthal mode number can be found for the DABM to be radially trapped. We find that higher $m$ DABMs are better localized, which is consistent with high- $m$ observations.
\end{abstract}

\section{Introduction}

Magnetospheric pulsations of the Pc4 and Pc5 types have periods on the order of $100 \mathrm{sec}$, and are often observed during a geomagnetic storm. Theoretical studies such as those by Chen and Hasegawa [3] etc. have identified the pulsations as drift Alfvén balloning mode instabilities excited via wave-particle interactions with resonant highly energetic protons. It was found that their growth rate is maximized for azimuthal numbers $m \approx 100$ [1] and for a field-aligned structure that is odd with respect to the equatcr. These results fit quite well with satellite observations such as those of Takahashi [4]. However, to the authors' knowledge, previous studies of these instabilities have been restricted to the one dimensional eigenvalue problem along the field line, i.e. no attempt was made to characterize the radial structure of these modes. It is obvious, that for the DABM to be a realistic instability candidate, it must be radially localized in the magnetosphere. This paper,

*Currently Dept. of Physics, University of California, Irvine, CA 
to the best of our knowledge, is the first that deals with these matters. As it will be shown, a plasma pressure gradient, like the one suggested by data in [5], could create a potential well in the radial direction which localizes the global Alfven-ballooning mode. In the picture developed here, the global mode suffers a small but finite damping due to absorption of the wave energy at the flux surface whose file line resonant frequency matches the global mode eigenfrequency. Both radial localization and damping depend on $m$ as well as the steepness of the pressure gradient. The damping rate is negligible for moderately high $m$.

\section{Theoretical framework}

In this paper we will be using a model equilibrium that is axisymmetric. The magnetohydrodynamic (MHD) momentum equations for the components $\eta_{\phi}, \eta_{\psi}$ of the perturbed perpendicular vector potential, were expressed in magnetic coordinates $(\phi, \chi, \psi)$ for the cold plasma case in [2,6]. For the hot plasma case they are the following:

$$
\begin{aligned}
D_{\phi} \eta_{\phi}-\frac{\kappa_{\psi}}{B_{0}^{2}}\left(p_{\perp}+p_{\|}\right) & =\left(\frac{\partial}{\partial \psi}-\beta \prime\right)\left(B_{\chi}+p_{\perp} / B_{0}^{2}\right), \\
D_{\psi} \eta_{\psi} & =-\frac{\partial\left(B_{\chi}+p_{\perp} / B_{0}^{2}\right)}{\partial \phi}
\end{aligned}
$$

and

$$
B_{\chi}=\frac{\partial \eta_{\psi}}{\partial \phi}-\frac{\partial \eta_{\phi}}{\partial \psi}
$$

where $\vec{B}_{0}=\vec{\nabla} \phi \times \vec{\nabla} \psi$ is the equilibrium magnetic field, $\beta \prime=2 \frac{\partial P_{0}}{\partial \psi} / B_{0}^{2}$, the perturbed electric field is $\vec{E}=-\partial_{t} \eta_{\phi} \vec{\nabla} \phi-\partial_{t} \eta_{\psi} \vec{\nabla} \psi, \phi$ is the azimuthal angle, $\psi$ the equilibrium flux function, $\chi$ is the coordinatc along $B_{0}\left(d \chi=B_{0} d l\right), l$ is the arc length on a flux surface, $B_{\chi}$ is the perturbed parallel magnetic field, $p_{\perp}$ and $p_{\|}$perturbed perpendicular and parallel pressures, $\kappa_{\psi}|\vec{\nabla} \psi|^{2}=\vec{\kappa} \cdot \vec{\nabla} \psi$ with $\vec{\kappa}$ the curvature of $\vec{B}_{0}, P_{0}$ is the equilibrium pressure which is assumed to be isotropic in this analysis, and 


$$
\begin{aligned}
& D_{\phi}=\frac{1}{B_{0}} \partial_{l} \frac{|\vec{\nabla} \phi|^{2}}{B_{0}} \partial_{l}+\frac{\omega^{2}|\vec{\nabla} \phi|^{2}}{v_{A}^{2} B_{0}^{2}}, \\
& D_{\psi}=\frac{1}{B_{0}} \partial_{l} \frac{|\vec{\nabla} \psi|^{2}}{B_{0}} \partial_{l}+\frac{\omega^{2}|\vec{\nabla} \psi|^{2}}{v_{A}^{2} B_{0}^{2}} .
\end{aligned}
$$

In general, $p_{\perp}$ and $p_{\|}$can contain contributions from trapped particles, wave-particle interactions and hydrodynamic terms as in [3]. We will include wave-particle interactions in a future and more detailed article, but for now we will assurne an isotropic and incompressible MHD fluid, which leads to $p_{\perp}=p_{\|}=-\eta_{\phi} \partial_{\psi} P_{0}$.

Also we define the following operator:

$$
E_{\phi}=D_{\phi}+2 \frac{\partial P_{0}}{\partial \psi} \frac{\kappa_{\psi}}{B_{0}^{2}}
$$

$D_{\psi}$ as well as $E_{\phi}$ act only on the $l$-dependence of their arguments, and they depend only parametrically on $\psi$. For each and every $\psi, D_{\psi}$ and $E_{\phi}$ have a complete set of eigenfunctions $\hat{\eta_{\phi}}(n, l, \psi), \hat{\eta_{\psi}}(n, l, \psi)$ along $l$ with eigenvalues $\omega_{\psi}^{2}(n, \psi)$ and $\omega_{\phi}^{2}(n, \psi)$ respectively, corresponding to the $n^{\text {th }}$ mode of each operator. In the present work we will consider only the case $n=1$, which corresponds to the lowest odd mode with respect to the equator, and suppress $n$ from now on. As expected, the solution to system Eqs. (1-3) depends critically on the $\omega_{\phi}^{2}$ and $\omega_{\psi}^{2}$ profiles which themselves depend on the equilibrium. The plasma equilibrium used in this paper was constructed in [1], by solving perturbatively the Grad-Shafranov equation with the plasma pressure profile $P(\psi)=P_{0}\left(1+\left(\frac{\psi-\psi_{0}}{\epsilon}\right)^{2 \alpha}\right)^{-1}$. Thus the self-consistent effect of a pressure gradient is only modeled locally and not globally. The equilibrium pressure profile employed is shown in Fig. 1, and the resulting $\omega_{\phi}^{2}$ and $\omega_{\psi}^{2}$ in Fig. 2 .

From Fig. 2 it is seen that $\omega_{\phi}^{2}(\psi)$ drops monotonically, as one moves away from the planet (i.e. $\psi$ decreases) until some local minimum $\omega_{\min }^{2}$, then rises until a local maximum $\omega_{\max }^{2}$ and subsequently drops monotonically again, as radial distance increases, tending to zero. If $\beta$ l is sufficiently high 


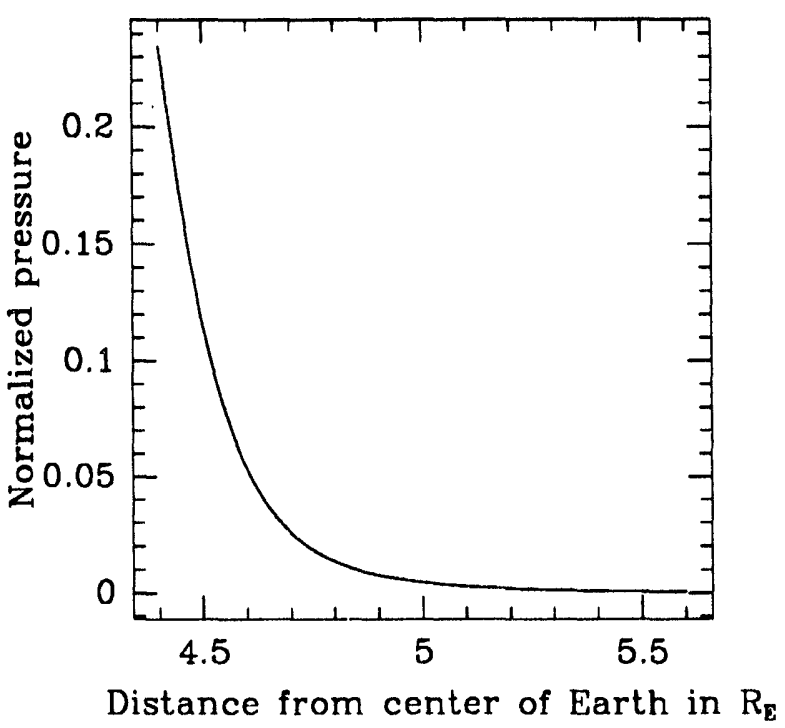

Figure 1: Pressure profile

$\omega_{\min }^{2}$ can become negative. $\omega_{\psi}^{2}(\psi)$ is always above $\omega_{\phi}^{2}(\psi)$ and monotonically dropping with increasing radial distance.

The operators $E_{\phi}$ and $D_{\psi}$ are self-adjoint because of the exclusion of wave-particle interactions, in the sense that $\int_{S}^{N} d l B_{0} \eta E_{\phi} \zeta=\int_{S}^{N} d l B_{0} \zeta E_{\phi} \eta$, with boundary conditions $\eta, \zeta=0$ at the boundary points, and similarly for $D_{\psi}$. Equations $1,2,3$ are not generally tractable in an analytic fashion. We adopt the ordering $\left|\frac{\partial}{\partial \phi}\right|=|m| \approx O\left(10^{2}\right)>>1$, which is consistent with observations, and we will be using $1 / m$ as an expansion parameter. Then we can emplny the WKB approximation in the radial dimension to reduce the two-dimensional problem to two nested 1D problems.

\section{WKB calculation}

We introduce the following WKB ansatz:

$$
\begin{aligned}
& \eta_{\phi}=\hat{\eta_{\phi}}(\psi, l) \exp m S(\psi), \\
& \eta_{\psi}=\hat{\eta_{\psi}}(\psi, l) \exp m S(\psi),
\end{aligned}
$$




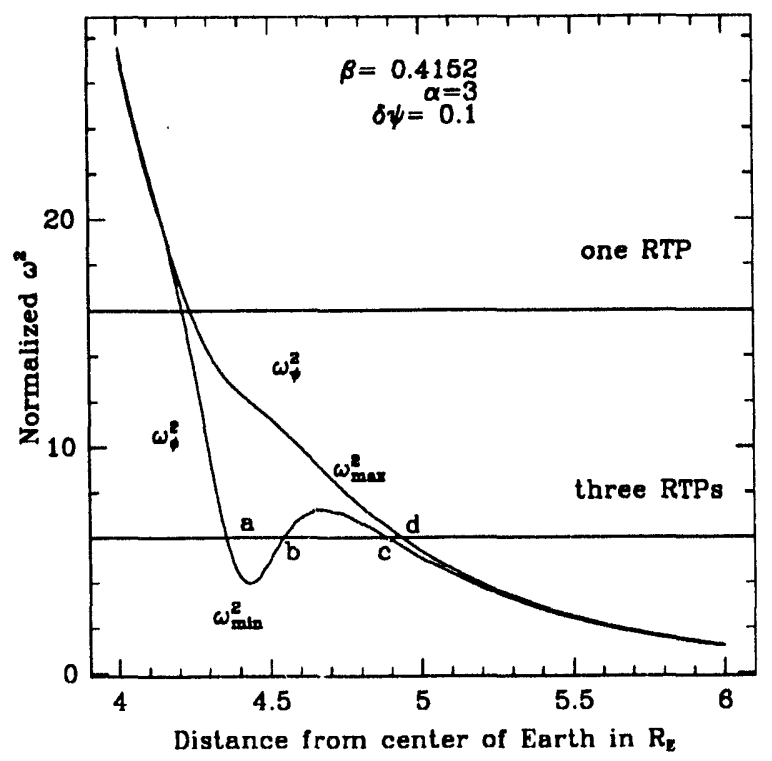

Figure 2: $\omega_{\phi}^{2}(\psi), \omega_{\psi}^{2}(\psi)$ profiles

$$
p_{\perp}=\hat{p_{\perp}}(\psi, l) \exp m S(\psi)
$$

Define $S_{0 \psi} \equiv \partial S_{0} / \partial \psi$. Introduce the expansion:

$$
\begin{aligned}
S & =S_{0}+\frac{1}{m} S_{1}+\frac{1}{m^{2}} S_{2}+\cdots, \\
\hat{\eta_{\phi}} & =\hat{\eta_{0 \phi}}+\frac{1}{m} \hat{\eta_{1 \phi}}+\frac{1}{m^{2}} \eta_{\hat{2} \phi}+\cdots, \\
\hat{\eta_{\psi}} & =\eta_{\hat{0} \psi}+\frac{1}{m} \eta_{\hat{1} \psi}+\frac{1}{m^{2}} \eta_{\hat{2} \psi}+\cdots
\end{aligned}
$$

With the WKB ordering that $S_{0 \psi}^{2}>>\frac{\partial S_{0 \psi}}{\partial \psi}$ and $S_{\psi} \hat{\eta}>>\frac{\partial \dot{\eta}}{\partial \psi}$, we have to lowest order:

$$
\left(S_{0 \psi}^{2} D_{\psi}-E_{\phi}\right) \hat{\eta_{0}}=0
$$

This is equivalent to $S_{0 \psi}^{2}=\hat{E}_{\phi} / \hat{D_{\psi}}$ with the definitions $\hat{E}_{\phi}=\left\langle\hat{\eta}_{0}, E_{\phi} \hat{\eta_{0}}\right\rangle, \hat{D_{\psi}}=\left\langle\hat{\eta}_{0}, D_{\psi} \hat{\eta_{0}}\right\rangle$. Here $\left\langle f_{1}, f_{2}\right\rangle=\int_{S}^{N} d l f_{1}(l) f_{2}(l) B_{0}$, that is, we integrate along the field line between its southern and northern boundary points. Equation (13) was derived using the boundary conditions that $\hat{\eta}_{0}=0$ at $l=l_{N}, l_{S}$, which are the points where the field line intersects the ionosphere. The ionosphere is 
assummed to be perfectly conducting. $S_{0 \psi}$ can be found as the eigenvalue of Eq. (13). In the next order exploiting the self-adjointness of $E_{\phi}$ and $D_{\psi}$ it can be shown that

$$
\left(\partial_{\psi} S_{0 \psi}+2 S_{0 \psi} S_{1 \psi}\right) \hat{D_{\psi}}+S_{0 \psi} \hat{R}=0
$$

where:

$$
\hat{R}=\left\langle\hat{\eta_{\phi}},\left(D_{\psi}\left(\partial_{\psi} \hat{\eta_{\phi \phi}}+\frac{\hat{p_{\perp} 0}}{B_{0}^{2}}\right)+\left(\partial_{\psi}-\beta \prime\right) D_{\psi} \hat{\eta}_{\hat{O} \phi}\right)\right\rangle
$$

\section{Solutions near turning points}

The WKB ansatz breaks down close to a $\psi$ where $S_{0 \psi}(\psi)$ becomes zero. This $\psi$ is termed a regular turning point (RTP). By inspection of Eq. (13), this happens when $E_{\phi}\left(l,{ }^{\prime \prime} / R T P ; \omega^{2}\right) \hat{\eta}_{0}\left(l, \psi_{R T P} ; \omega^{2}\right)=$ 0 or equivalently, when $\hat{\eta}_{0}\left(l, \psi_{R T P}\right)=\hat{\eta}_{\phi}\left(l, \psi_{R T P}\right)$ and $\omega^{2}=\omega_{\phi}^{2}\left(\psi_{R T P}\right)$. Another type of turning point is the singular one, encountered if for some $\psi$ there is no finite eigenvalue $S_{0 \psi}$ for Eq. (13). From Eq. (13) this singular turning point (STP), occurs when $\hat{D_{\psi}}=0$ or equivalently when $\hat{\eta}_{0}\left(l, \psi_{S T P}\right)=$ $\eta_{\psi}\left(l, \hat{\psi}_{S T P}\right)$ and $\omega^{2}=\omega_{\psi}^{2}\left(\psi_{S T P}\right)$. The singular turning point corresponds pnysically to the resonant layer where the wave energy could be dissipated.

To derive the connection formulas near $\psi_{R T P}$ we use a multiscale expansion of the system Eqs. (13 ), which involves a fast and a slow $\psi$ dependence, and an expansion in powers of $m^{-2 / 3}$. Define $\delta=\psi-\psi_{R T P}$ and $E_{\phi}^{(0)}=E_{\phi}\left(\psi_{R T P}\right)$. Then to lowest order:

$$
E_{\phi}^{(0)} \eta_{0}=0
$$

which is the regular turning point condition. Eq. (16) indicatss that $\eta_{0}$ is separable in $l$ and $\delta$, namely, $\eta_{0}=\hat{\eta_{\phi}}\left(l, \psi_{R T P}\right) h(\delta)$. Using the self adjointniss of $E_{\phi}^{(0)}$ we get from the next order:

$$
\frac{\partial^{2} h}{\partial \delta^{2}}=h \delta \frac{\left\langle\hat{\eta_{\phi}}, E_{\phi}^{(1)} \hat{\eta_{\phi}}\right\rangle}{\left\langle\hat{\eta_{\phi}}, D_{\psi}^{(0)} \hat{\eta_{\phi}}\right\rangle}
$$

where $E_{\phi}^{(1)}$, and $D_{\psi}^{(0)}$ correspond to $\frac{\partial E_{\phi}}{\partial \psi}$, and $D_{\psi}$ respectively, evaluated at $\psi_{R T P}$. Eq. (17) is exactly the Airy equation for the $\psi$ dependence of the global mode near $\psi_{R T P}$. The WKB connection 
formulae can be derived by asymptotically matching the solutions of Eq. (17) to the WKB solutions given in the preceding section. Thus we have shown that by employing the WKB method a two dimensional eigenvalue problem can be reduced to two nested one dimensional problems. We could carry out a similar analysis near the STP but this is not necessary as will be discussed later on.

\section{The Dispersion Relation}

By referring to Fig. 2 and remembering that $S_{0 \psi}=0$ when $\omega^{2}=\omega_{\phi}^{2}$, we can identify the possibilities that exist for the regular turning points, and similarly for the singular turning point. In the figure, we see that depending on $\omega^{2}$ we have the following cases:

1. $\omega^{2}>\omega_{\max }^{2}$ gives one regular turning point,

2. $\omega_{\max }^{2}>\omega^{2}>\max \left(0, \omega_{\min }^{2}\right)$ gives three regular turning points,

3. For large $\beta \prime$ we have the possibility $\omega_{\min }^{2}<\omega^{2}<0$ (i.e. local balooning unstable mode) and we obtain two RTP's,

4. $0<\omega^{2}<\omega_{\min }^{2}$ we get one RTP.

There is always a singular turning point at $\omega^{2}=\omega_{\psi}^{2}\left(\psi_{S T P}\right)$.

We recall from Eq. (16) $\left(S_{0 \psi}^{2}=\frac{E_{\phi}}{\vec{D}_{\psi}}\right)$ that for $\omega^{2} \neq \omega_{\phi}^{2}(\psi), \hat{E}_{\phi}(\psi)$ will be nonzero. The same can be said for $\omega_{\psi}^{2}$ and $\hat{D_{\psi}}$. Writing out explicitly $\hat{E_{\phi}}$ we get :

$$
\hat{E}_{\phi}=\int_{S}^{N} d l \frac{1}{B_{0}^{2}}\left(\left(\kappa_{\psi} \beta l+\frac{\omega^{2}|\vec{\nabla} \phi|^{2}}{v_{A}^{2}}\right){\hat{\eta_{0}}}^{2}-\left|\partial_{l}{\hat{\eta_{0}}}^{2}\right|^{2}|\vec{\nabla} \phi|^{2}\right) .
$$

The above equation is the variational problem from which $E_{\phi} \hat{\eta}_{\phi}=0$ can be derived, and thus it is clear that $\hat{E}_{\phi}(\psi)<0$ for $\omega^{2}<\omega_{\phi}^{2}(\psi)$. Similarly $\hat{D_{\psi}}<0$ for $\omega^{2}<\omega_{\psi}^{2}$. Assuming that $\frac{\partial \omega_{\phi}^{2}}{\partial \psi} \neq 0$ at $\psi=\psi_{R T P}$ and noting that $\omega_{\phi}^{2}<\omega_{\psi}^{2}$ we can conclude that:

1. $S_{0 \psi}^{2}(\psi)>0$ for $\omega^{2}<\omega_{\phi}^{2}(\psi)<\omega_{\psi}^{2}(\psi)$, 
2. $S_{0 \psi}^{2}(\psi)<0$ for $\omega_{\phi}^{2}(\psi)<\omega^{2}<\omega_{\psi}^{2}(\psi)$,

3. $S_{0 \psi}^{2}(\psi)>0$ for $\omega_{\phi}^{2}(\psi)<\omega_{\psi}^{2}(\psi)<\omega^{2}$.

To have possible radial localization we need to have negative $S_{0 \psi}^{2}$ between two RTP's, and according to the above arguments this can happen only if $\max \left(\omega_{\min }^{2}, 0\right)<\omega^{2}<\omega_{\max }^{2}$. The case of $\omega_{\min }^{2}<\omega^{2}<0$ could also fulfill this condition, but it corresponds to ideal MHD instabilities, which require higher drive and is beyond the scope of the present analysis. Thus, we have to look for the global eigenvalue $\omega^{2}$ between these two limits. It is possible, in principle, that the $\omega_{\phi}^{2}$-well could become so shallow that there will be only one regular turning point in all cases and thus no mode could be localized there. That could happen if $\beta \prime$ is below a certain threshold.

To completely specify the problem, we need to impose boundary conditions. Referring to the previous results and Fig. 2, we see that, to the left of the RTP $a$ the WKB solution will be a sum of an exponentially growing and an exponentially decaying part. The appropriate boundary condition in this region is that the solution be spatially decaying. The other boundary condition is supplied by the existence of the resonant layer (STP) at $d$. In the MHD description the mode energy will be completely absorbed there, and so we know that there cannot be a reflected wave in the region between $c$ and $d$. This corresponds to an outgoing wave boundary condition.

Proceeding as in the usual one-dimensional case, we connect the various WKB solutions through the turning points and impose the relevant boundary conditions. We then obtain the following WKB eigenvalue condition or dispersion relation:

$$
\sin \left(m I_{a b}(\omega)+\frac{\pi}{2}\right)+\frac{i}{4} \exp (-2 m T(\omega)) \cos \left(m I_{a b}(\omega)+\frac{\pi}{2}\right)=0,
$$

where $I_{a b}=\int_{a}^{b} d \psi \sqrt{-S_{0 \psi}^{2}}$ and $T=\int_{b}^{c} d \psi S_{0 \psi}$, with $a, b$ being the two RTP's defining the localization area, and $b, c$ defining the tunnelling region. Note that, assumming total wave absorption, the exact location of the resonant layer does not enter in our results. 
Assuming that $\omega=\omega_{r}+i \gamma$ and that $\gamma \ll \omega_{r}$, we get

$$
\begin{gathered}
I_{\mathrm{a} b}\left(\omega_{r}\right)=\frac{\left(r+\frac{1}{2}\right)}{m} \pi \\
\gamma=-\frac{\exp \left(-2 m T\left(\omega_{r}\right)\right)}{4 m \frac{\partial I_{a b}\left(\omega_{r}\right)}{\partial \omega_{r}}}
\end{gathered}
$$

In Eq. (20) $r$ is the radial 'quantum' number, the number of radial nodes of the global mode between the turning points $a$ and $b$. Equation (21) shows that the damping rate is controlled by the exponential tunnelling factor. That means that the least damped modes are those that have large $\mathrm{T}$, or, equivalently, those localized close to the bottom of the well. However we can see from the quantization condition that the larger $m$ is, the smaller $I_{a b}$ has to be, so that we have to have the corresponding eigenfrequencies closer to $\omega_{\min }^{2}$. At the same time $\exp (-2 m T)$ becomes very small too, justifying the assumption $\gamma \ll \omega_{r}$ for $m \gg 1$.

Physically, higher $m$ modes have to tunnel their way for a longer distance, and thus by the time they couple to the field line resonance, there is very little energy to be dissipated. Note that in the present work, we have assummed first harmonic antisymmetric modes; i.e. modes with only one node. For higher longitudinal mode numbers, we would simply have to find a higher eigenmode of Eq. (13) with a corresponding $S_{0 \psi}^{2}$ eigenvalue.

We can predict which is the lowest $m$ number a mode can have and still be expected to be radially localized and not leak out readily towards the shear Alfvén continuous spectrum. We can calculate $I_{a b}\left(\omega_{r}=\omega_{\max }\right)$ and use the fact that $\left(r+\frac{1}{2}\right) / m<I_{a b}\left(\omega_{\max }\right)$ to get the lower limit on $m$. An approximate upper limit for the observable $m$ can be found by looking at the growth rates due to the resonant particle interactions, which show a clear peak [1]. Figure 3 shows $\omega$ and $\gamma$ for various $m$ numbers, assuming one radial node. Note that as $m$ becomes smaller, the damping rate increases sharply due to the decreasing tunnelling loss. From Eq. (20) we can also see that higher $r$ numbers require, higher $m_{\min }$ modes, which however are not easily excited by wave-particle interactions. 
This means that effectively only $r=0,1$ ( radial nodes) are viable candidates. Also modes with higher longitudinal mode numbers $(n)$, are less likely to be localized. The reason for this is that, for high $n$ the effect of the ballooning term in $E_{\phi}$ is very diminished and the well will become shallow or disappear altogether. Interestingly enough the lowest $n$ is favored by the excitation mechanism too.
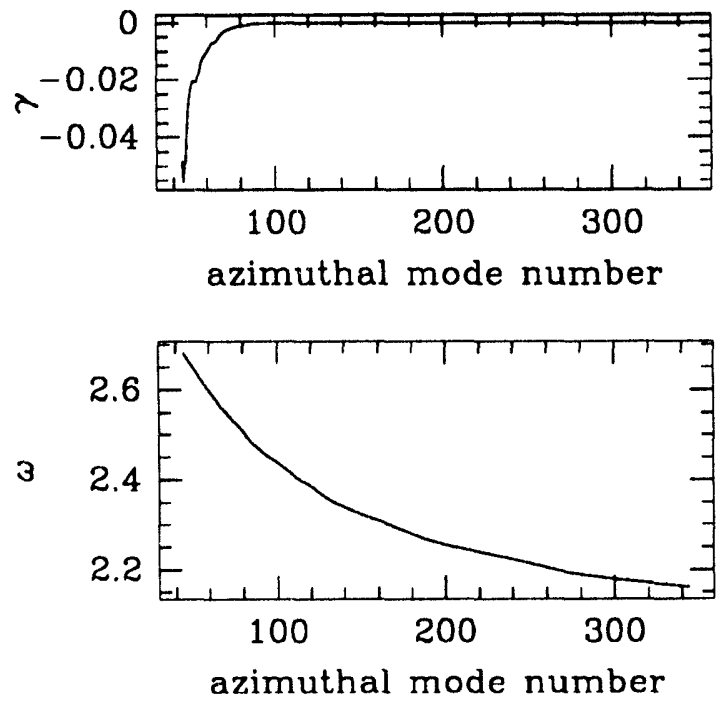

Figure 3: $\omega$ and $\gamma$ versus azimuthal mode number

\section{Summary and Discussion}

We have shown that the Drift Alfvén Ballooning modes, previously studied locally along the field line, can be radially localized in the presence of a steep enough pressure gradient which almost vanishes after a point. The radial extent of localization is defined essentially by the locations of the maximum and the minimums of the pressure gradient as a function of radial distance. These modes, however, suffer finite damping through their coupling to field line resonances. It is important to note that the damping is negligible for $m \approx 100$ and longitudinal "quantum number" $n=1$, (odd symmetry with respect to the equator). These numbers correspond to modes with peak growth rates, 
as well as to those observed by satellites. The present results thus further support the DABM as a viable instability candidate. Observing the radial structure of Pc4 and Pc5 waves in the terrestial magnetosphere has always been difficult given that most satellites are in geosynchronous orbits. The predictions of this work thus, might not be easily testable even though they are consistent with satellite observations. Finally, we note that the present approach can be readily extended to include effects of wave-particle interactions as well as pressure anisotropy. The results will be reported in a more detailed future publication.

Acknowledgements This work is supported by NSF Grant ATM-9396158 and U.S. D.o.E. Contract No. DE-AC02-76-CHO-3073.

\section{References}

[1] Anthony Chan. Interaction of Energetic Ring Current Protons with Magnetospheric Hydromagnetic Waves. PhD thesis, Princeton University, 1991.

[2] Liu Chen and Steven C. Cowley. On field line resonances of hydromagnetic alfven waves in dipole magnetic field. Geophysical Research Letters, 16(8):895-897, August 1989.

[3] Liu Chen and Akira Hasegawa. Kinetic theory of geomagnetic pulsations 1. Internal excitations by energetic particles. Journal of Geophysical Research, 96:1503-1512, 1991.

[4] Takahashi K. Multisatellite studies of ULF waves. Advances in Space Research, 8:427, 1988.

[5] S. M. Krimigis, G. Gloeckler, R. M. McEntire, T. A. Potemra, F. L. Scarf, and E. G. Shelley. Magnetic storm of September 4,1984: A synthesis of ring current spectra and energy densities measured with AMPTE/CCE. Geophysical Reseanch Letters, 12:329, 1985.

[6] H. R. Radoski. A note on oscillating field lines. Geophysical Research Letters, 72:418, 1967. 


\section{EXTERNAL DISTRIBUTION IN ADDITION TO UC-420}

Dr. F. Paoloni, Univ. of Wollongong, AUSTRALIA

Prof. M.H. Brennan, Univ. of Sydney, AUSTRALIA Plasma Research Lab., Australian Nat. Univ., AUSTRALIA

Prof. I.R. Jones, Flinders Univ, AUSTRALIA

Prof. F. Cap, inst. for Theoretical Physics, AUSTRIA

Prof. M. Heindler, Institut für Theoretische Physik, AUSTRIA

Prof. M. Goossens, Astronomisch Instituut, BELGIUM

Ecole Royale Miliaire, Lab. de Phy. Plasmas, BELGIUM

Commission-European, DG. XII-Fusion Prog., BELGIUM

Prof. R. Bouciqué, Rijksuniversiteit Gent, BELGIUM

Dr. P.H. Sakanaka, Instituto Fisica, BRAZIL

Prof. Dr. I.C. Nascimento, Instituto Fisica, Sao Paulo, BRAZIL Instituto Nacional De Pesquisas Espaciais-INPE, BRAZIL Documents Office, Atomic Energy of Canada Lid., CANADA Ms. M. Morin, CCFWTokamak de Varennes, CANADA Dr. M.P. Bachynski, MPB Technologies, Inc, CANADA Dr. H.M. Skarsgard, Univ. of Saskatchewan, CANADA Prof. J. Teichmann, Univ. of Montreal, CANADA Prof. S.R. Sreenivasan, Univ. of Calgary, CANADA Prof. T.W. Johnston, INRS-Energie, CANADA Dr. R. Bolton, Centre canadien de fusion magnétique, CANADA Dr. C.R. James,, Univ. of Alberta, CANADA Dr. P. Lukác, Komenského Universzita, CZECHO-SLOVAKIA The Librarian, Culham Laboratory, ENGLAND Library, R61, Rutherford Appleton Laboratory, ENGLAND Mrs. S.A. Hutchinson, JET Library, ENGLAND Dr. S.C. Sharma, Univ. of South Pacific, FIJI iSLANDS P. Mähönen, Univ. of Helsinki, FINLAND Prof. M.N. Bussac, Ecole Polytechnique, FRANCE C. Mouttet, Lab. de Physique des Milieux Ionisés, FRANCE J. Radet, CEN/CADARACHE - Bat 506, FRANCE Prof. E. Economou, Univ. of Crete, GREECE Ms. C. Rinni, Univ. of loannina, GREECE Preprint Library, Hungarian Academy of Sci., HUNGARY

Dr. 8. DasGupta, Saha Inst. of Nuclear Physics, INDIA

Dr. P. Kaw, Inst. for Plasma Research, INDIA

Dr. P. Rosenau, Israel Inst. of Technology, ISRAEL Librarian, International Center for Theo Physics, ITALY Miss C. De Palo, Associazione EURATOM-ENEA , ITALY Dr. G. Grosso, Istituto di Fisica del Plasma, ITALY Prof. G. Rostangni, Istituto Gas lonizzati Del Cnr, ITALY
Dr. H. Yamato, Toshiba Res \& Devel Center, JAPAN

Prof. I. Kawakami, Hiroshima Univ., JAPAN

Prof. K. Nishikawa, Hiroshima Univ., JAPAN

Librarian, Naka Fusion Research Establishment, JAERI, JAPAN

Director, Japan Atomic Energy Research Inst., JAPAN

Prot. S. Itoh, Kyushu Univ., JAPAN

Research Info. Ctr., National Instit. for Fusion Science, JAPAN

Prof. S. Tanaka, Kyoto Univ., JAPAN

Library, Kyoto Univ., JAPAN

Prot. N. Inoue, Univ. of Tokyo, JAPAN

Secretary, Plasma Section, Electrotechnical Lab., JAPAN

S. Mori, Technical Advisor, JAERI, JAPAN

Dr. O. Mitarai, Kumamoto Inst. of Technology, JAPAN

Dr. G.S. Lee, Korea Basic Sci. Ctr., KOREA

J. Hyeon-Sook, Korea Atomic Energy Research Inst., KOREA

D.I. Choi, The Korea Adv. Inst. of Sci. \& Tech., KOREA

Prof. B.S. Liley, Univ. of Waikato, NEW ZEALAND Inst of Physics, Chinese Acad Sci PEOPLE'S REP. OF CHINA Library, Inst. of Plasma Physics, PEOPLES REP. OF CHINA Tsinghua Univ. Library, PEOPLE'S REPUBLIC OF CHINA Z. Li, S.W. Inst Physics, PEOPLE'S REPUBLIC OF CHINA Prof. J.A.C. Cabral, Instituto Superior Tecnico, PORTUGAL Prof. M.A. Hellberg, Univ. of Natal, S. AFRICA. Prof. D.E. Kim, Pohang Inst. of Sci. \& Tech., SO. KOREA Prof. C.I.E.M.A.T, Fusion Division Library, SPAIN

Dr. L. Stenflo, Univ. of UMEA, SWEDEN Library, Royal Inst. of Téchnology, SWEDEN Prof. H. Wilhelmson, Chalmers Univ. of Tech., SWEDEN Centre Phys. Des Plasmas, Ecole Polytech, SWITZERLAND Bibliotheek, Inst. Voor Plasma-Fysica, THE NETHERLANDS Asst. Prof. Dr. S. Cakir, Middle East Tech. Univ., TURKEY Dr. V.A. Glukhikh,Sci. Res. Inst. Electrophys.l Apparatus, USSR Dr. D.D. Ryutov, Siberian Branch of Academy of Sci., USSR Dr. G.A. Eliseev, I.V. Kurchatov Inst., USSR Librarian, The Ukr.SSR Academy of Sciences, USSR Dr. L.M. Kovrizhnykh, Inst. of General Physics, USSR Kernforschungsanlage GmbH, Zentralbibliothek, W. GERMANY Bibliothek, Inst. Für Plasmaforschung, W. GERMANY Prof. K. Schindler, Ruhr-Universitát Bochum, W. GERMANY Dr. F. Wagner, (ASDEX), Max-Planck-Institut, W. GERMANY Librarian, Max-Planck-Institut, W. GERMANY 

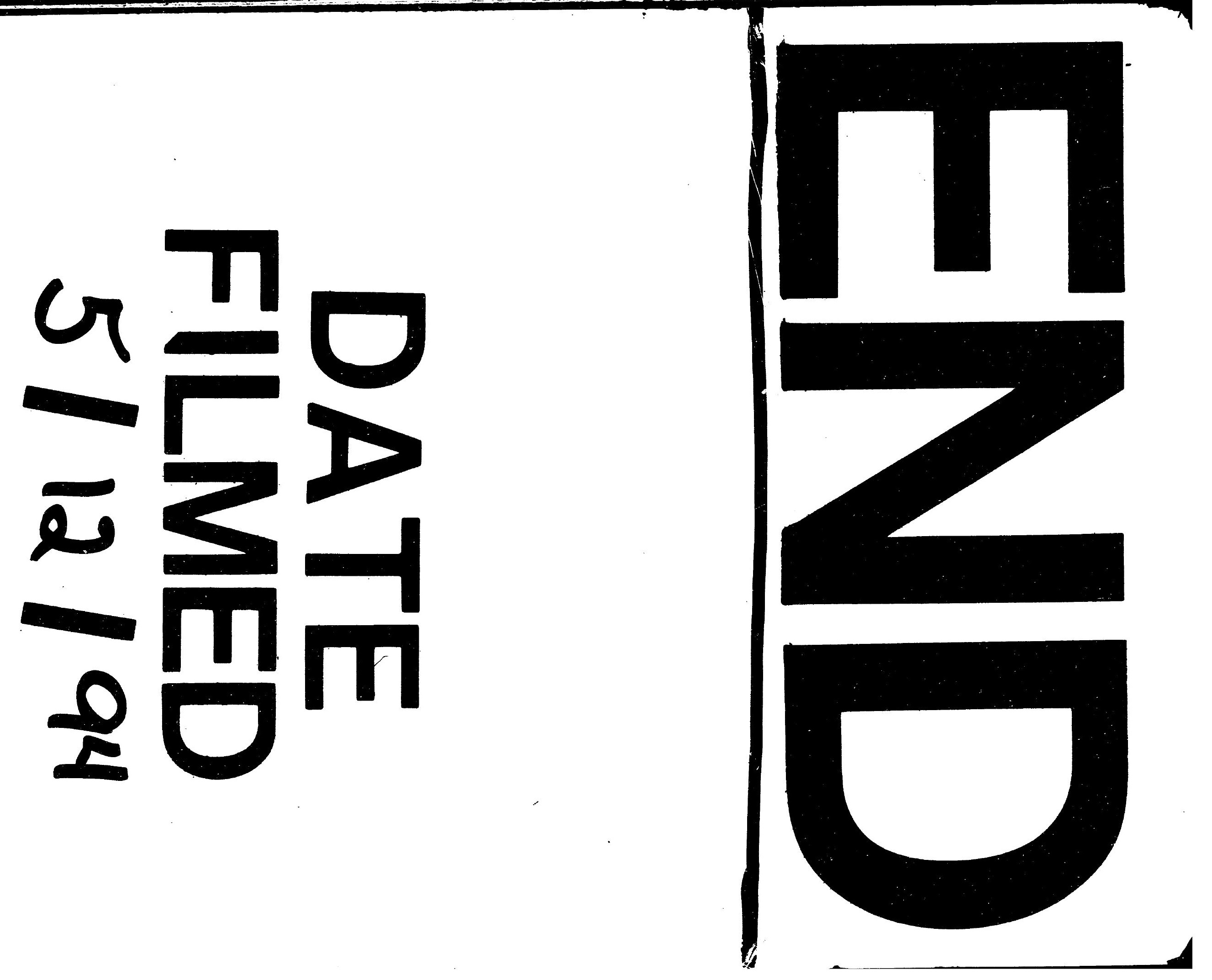
